\title{
Não tratamos a congestão pulmonar e sistêmica na insuficiência cardíaca aguda adequadamente
}

Felipe Liger Moreira ${ }^{1 *}$, Geovane Wiebelling da Silva ${ }^{1}$

1 Hospital das Clínicas da Faculdade de Medicina da Universidade de São Paulo, São Paulo, Brasil.

* Autor correspondente. Endereço de e-mail: ligermfelipe@hotmail.com

\section{RESUMO}

A insuficiência cardíaca aguda é uma doença atual, sendo de grande relevância para o sistema de saúde público e privado. As evidências disponíveis demonstram a diminuta aplicação de medidas comprovadamente eficazes, ora condicionadas pelo limitado conhecimento sobre aspectos pouco abordados da doença, sobretudo em publicações para medicina de emergência. Essa revisão será narrativa e focará em reunir as melhores informações para realização do diagnóstico de insuficiência cardíaca no departamento de emergência, avaliação integrada da congestão e fase vulnerável.

Palavras-chave: Insuficiência Cardíaca, Insuficiência Cardíaca Aguda, IC, Departamento de Emergência.

\section{Introdução}

Insuficiência cardíaca (IC) é uma síndrome clínica com incapacidade de gerar débito cardíaco que atenda às necessidades teciduais e orgânicas e/ou o faz às custas de elevação das pressões de enchimento intracardíacas com diferentes causas e fisiopatologias mais que uma doença específica, sendo desprovida de qualquer sinal patognomônico único, seja histológico ou bioquímico, e reconhecida como possível resultado final de muitos estados de doença bastante distintos e variados, comumente associada a doença arterial coronariana, hipertensão arterial sistêmica, diabetes mellitus e doença renal crônica [1]. O termo "insuficiência cardíaca crônica " reflete a natureza progressiva e persistente da doença, enquanto o termo "insuficiência cardíaca aguda " fica reservado para mudanças rápidas e/ou graduais de sinais ou sintomas necessitando de tratamento imediato [1]. Os sinais e sintomas podem ocorrer em indivíduos sem qualquer diagnóstico prévio de IC, quando será denominada IC aguda de novo ou nova $-20 \%$, ou podem ocorrer em indivíduos com diagnóstico de IC crônica, quando será denominada IC aguda crônica descompensada - 80\% [2]. Essa classificação tem implicação clínica relevante, já que os pacientes com IC aguda de novo apresentam maior mortalidade intra-hospitalar, mas menor mortalidade após a alta e menor risco de readmissão [2,3]. O diagnóstico no departamento de emergência pode ser uma tarefa desafiadora, sobretudo em pacientes que compartilham outras justificativas para os sinais e sintomas, como, por exemplo, os pacientes com doença pulmonar obstrutiva crônica (DPOC). Com intuito de unificar o diagnóstico de IC fornecendo um critério prático, acurado e de fácil reprodutibilidade, a European Society of Cardiology (ESC) publicou em 2021 a primeira definição universal de IC, que será utilizada nesta revisão quando abordarmos o diagnóstico no departamento de emergência [3].

IC aguda é a principal responsável por admissão hospitalar não planejada em pessoas com $>65$ anos de idade, com alta morbidade e mortalidade intra-hospitalar e frequente reinternação [2]. Independente da fração de ejeção do ventrículo esquerdo (FEVE), a sobrevida média após hospitalização de pacientes com IC aguda é de 2 anos, com a fase de maior mortalidade sendo aquela compreendida entre os primeiros meses imediatamente após a alta hospitalar [2, 4]. Nos países desenvolvidos, se tornou um problema de saúde pública, afetando $2 \%$ da população adulta, com o triplo de internações hospitalares quando 
comparado a 1990. Contrapondo a evolução do tratamento de insuficiência cardíaca crônica de fração de ejeção reduzida (ICFER), que modificou a história natural da doença, a IC aguda é ainda associada com desfechos inaceitáveis. Estima-se que 1 em cada 6 pacientes internados morrerá em até 30 dias da hospitalização e, inaceitavelmente, uma taxa de readmissão hospitalar de até $24 \%$ em 30 dias e $50 \%$ em 6 meses. O primeiro registro brasileiro de IC aguda publicado em 2015 - BREATHE - incluiu 1.263 pacientes em 51 centros de diferentes regiões do país e clarificou que a IC é um problema de magnitude epidêmica e em progressão [5]. Os pacientes apresentavam alta taxa de mortalidade intrahospitalar (13\%) e baixa taxa de prescrição de terapias baseadas em evidência. Nestes registros, a mortalidade em 1 ano de IC aguda foi de até $17 \%$, e a necessidade de reinternação ocorreu em até $66 \%$ dos casos. O estudo INTER-CHF, um dos maiores registros de IC em regiões de baixa a média renda no mundo, acompanhou 5.823 pacientes com IC de 108 centros em seis regiões geográficas. A mortalidade geral em 1 ano foi de $16,5 \%$, com a maior mortalidade na África (34\%) e na Índia (23\%), contrapondo a mortalidade média no sudeste da Ásia (15\%) e a menor mortalidade na China (7\%), América do Sul (9\%) e Oriente Médio (9\%)[6] .

Os pacientes com IC aguda crônica descompensada ao se apresentarem no departamento de emergência em geral não possuem perda adicional de função cardíaca , já os pacientes com IC aguda quase sempre se apresentam em contexto de insulto levando a perda de função, como , por exemplo, os casos secundários a infarto agudo do miocárdio, miocardite fulminante e insuficiência valvar aguda grave[1,2]. Aproximadamente $90 \%$ dos pacientes que se apresentam no departamento de emergência com IC aguda são caracterizados com fenótipo de congestão sem hipoperfusão [1]. Hipoperfusão define choque cardiogênico, a forma mais grave de apresentação, representando $10 \%$ dos casos e associado com mortalidade intra-hospitalar 5 a 10 vezes maior quando comparado aos casos normoperfundidos[4]. Entretanto, o padrão de congestão e a sua fisiopatologia difere entre os casos de IC aguda crônica descompensada e IC aguda de novo [1]. A congestão não é apenas a principal causa para hospitalização em pacientes com IC aguda $[1,2,4]$, mas também é a principal causa de reinternação durante os primeiros meses após a alta e está associada ao aumento de mortalidade. Quando os sinais e/ou sintomas de congestão melhoram, se observa ainda significativa congestão hemodinâmica, que pode anteceder em semanas a fase inexoravelmente sintomática [7]. Entretanto, este conhecimento é pouco difundido e reconhecido, induzindo ao equívoco de avaliação simplória e baseada em resolução de sintomas. É importante ressaltar que as ferramentas mais utilizadas atualmente para avaliação de congestão pulmonar e/ou sistêmica são desprovidas de sensibilidade (exame físico e radiografia de tórax) para identificação de sua fase assintomática [8]. Adicionalmente, não há nenhuma ferramenta validada e universalmente aceita para identificação de pacientes de maior risco que se apresentam com fenótipo de congestão [8]. A ultrassonografia point of care (POCUS) apresenta maior acurácia para diagnóstico de IC aguda por prover maior sensibilidade e especificidade para detecção de congestão pulmonar e/ou sistêmica de causa cardíaca, também servindo como ferramenta para avaliação dinâmica durante a terapia instituída no departamento de emergência, estando os seus achados associados a mortalidade $[8,9,10]$. Diferentemente do entendimento comum, os estudos demonstram que o período de maior vulnerabilidade, ou seja, de maior risco de eventos adversos incluindo morte, é aquele compreendido imediatamente após a alta hospitalar [7]. A relação indissociável entre congestão residual e risco de reinternação e mortalidade exige uma mudança imediata do cuidado do paciente com IC aguda no departamento de emergência englobando o conhecimento que vai além de terapia com diuréticos e vasodilatadores condicionada pela classificação clínico hemodinâmica. Esta revisão focará em estabelecer as bases para o diagnóstico acurado de IC aguda integrado ao conhecimento atual sobre congestão e fase vulnerável. As doenças cardiovasculares se tornaram a principal causa de morte em países desenvolvidos e em desenvolvimento ${ }^{1-4}$ tornando-se um problema de saúde global com proporções epidêmicas.

\section{Diagnóstico de IC no Departamento de Emergência}

O início da avaliação coordenada de um paciente com IC aguda começa com o seu reconhecimento. Estudos randomizados e controlados mostram que o atraso ou a perda do diagnóstico está intimamente relacionado ao aumento do custo do cuidado, maior tempo de permanência no hospital, maior necessidade de internação em 
unidade de terapia intensiva, menor nível de satisfação do paciente e maior mortalidade $[3,8,9,10,11]$. Adicionalmente, um estudo observacional demonstrou que os pacientes que recebiam terapia diurética em menos de uma hora da chegada no departamento de emergência apresentavam menor mortalidade [12]. IC é uma doença heterogênea que não apresenta um método diagnóstico padrão-ouro [3]. Diferentes definições foram desenvolvidas ao longo do tempo e com diferentes propostas, variando desde definições de "livros didáticos", que são tipicamente focadas na fisiopatologia, até definições de caso, como os critérios de Framingham, que são usados principalmente em pesquisas clínicas [3]. 'IC é uma doença complexa, na qual o coração é incapaz de bombear sangue de forma a atender às necessidades metabólicas teciduais, ou pode fazê-lo somente com elevadas pressões de enchimento' é uma definição pouco prática e de difícil documentação na rotina do departamento de emergência. Ainda assim, um estudo de pacientes com IC avançada esperando implantação de dispositivo de assistência ventricular esquerda demonstrou que apenas $25 \%$ dos pacientes apresentavam débito cardíaco insuficiente para as demandas metabólicas teciduais. Há inúmeras condições que podem erroneamente ser definida como IC ou coexistir, já que os sintomas e/ou sinais não são exclusivos da doença, não sendo uma tarefa fácil realizar essa diferenciação. Os sintomas e/ou sinais de IC podem ser típicos ou menos típicos e mais específicos ou menos específicos, respectivamente (Tabela 1)[3]. Apesar de alguns sintomas e/ou sinais poderem representar estado de baixo débito cardíaco ou serem comumente encontrados em contexto de IC direita ou biventricular, não são definidores de tais contextos. Diferentemente da conclusão natural que muitos acabam desenvolvendo, o ecocardiograma não realiza diagnóstico de IC. O mais forte argumento para usar a FEVE em contexto de IC é para definição da estratégia de tratamento e prognóstico, mas não para diagnóstico [3]. O grupo de IC com FEVE reduzida apresenta terapia que altera a história natural da doença de acordo com estudos randomizados e controlados, diferentemente da população com IC de FEVE preservada, que ainda não possui terapia que altere a história natural da doença $[1,3]$.

A European Society of Cardiology (ESC) publicou em 2021 uma definição universal de IC que é simples, mas conceitual e concordante com os elementos em comum das diversas definições de

outras entidades, com aplicabilidade majoritariamente universal, validade prognóstica e terapêutica, sensibilidade e especificidade aceitável.

Sintomas e/ou sinais de IC causados por anormalidade cardíaca estrutural e/ou funcional, corroborada por no mínimo um dos seguintes: peptídeos natriuréticos elevados ou evidência objetiva de congestão pulmonar ou sistêmica (Figura 1).

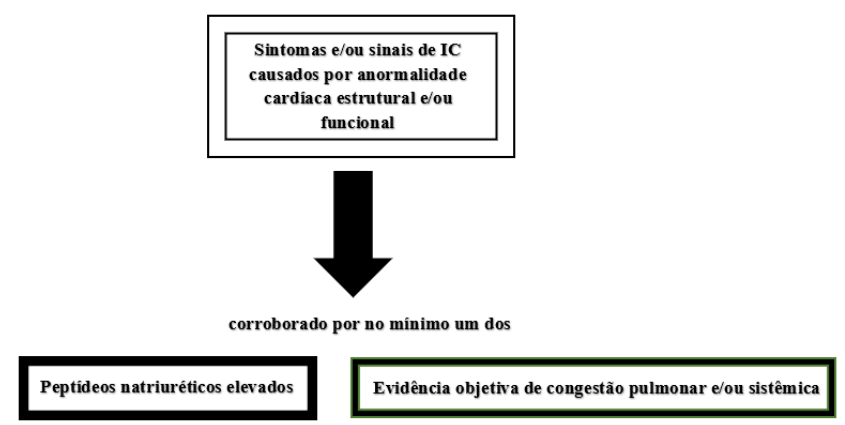

Figura 1. Adaptado de Bozkurt et al. Universal definition and classification of heart failure: a report of the Heart Failure Society of America, Heart Failure Association of the European Society of Cardiology, Japanese Heart Failure Society and Writing Committee of the Universal Definition of Heart Failure European Journal of Heart Failure (2021)

\begin{tabular}{|c|c|}
\hline \multicolumn{2}{|c|}{ Sintomas de insuficiência caríaca } \\
\hline Típicos & $\begin{array}{l}\text { Dispnéia } \\
\text { Ortopnéia * } \\
\text { Dispnéia paroxística noturna * } \\
\text { Tolerância reduzida ao esforço * } \\
\text { Fadiga ** } \\
\text { Edema de outras partes do corpo que o torn } \\
\text { Bendopneia }\end{array}$ \\
\hline Menos típicos & $\begin{array}{l}\text { Tosse noturna } \\
\text { Sibilância } \\
\text { Empachamento/ Plenitude pós-prandial † } \\
\text { Saciedade precoce † } \\
\text { Perda do apetite } \\
\text { Declínio na função cognitiva, confusão } \\
\text { (especialmente em idosos ) } \\
\text { Depressão } \\
\text { Tontura, síncope ** }\end{array}$ \\
\hline
\end{tabular}

Sinais de insuficiência cardíaca

Mais específicos

Menos específicos
Pressão venosa jugular elevada * $\dagger$ B3 *

Presença de galope associado com B3 e B4 Cardiomegalia, ictus cordis deslocado lateralmente Reflexo hepatojugular Respiração de Cheyne-Stokes em IC ] avançada **

Edema periférico (tornozelo, sacral, escrotal) Estertores pulmonares *

Ganho de peso não intencional 
(> 2kg/semana)

Perda de peso (em IC avançada) com sarcopenia e caquexia

Sopro cardíaco

Redução do murmúrio vesicular e macicez à percussão das bases pulmonares sugerindo derrame pleural

Taquicardia, pulso irregular

Taquipneia

Hepatomegalia/ascite

Extremidades frias **

Oligúria **

Pressão de pulso estreita **

* Comumente utilizado em estudos clínicos, registros, scores de risco e validado por avaliação da sensibilidade e especificidade

** Comum em estado de baixo débito cardíaco

† Pode ser típico em contexto de IC direita ou biventricular

Tabela 1. Sintomas e sinais de insuficiência cardíaca. Adaptado de Bozkurt et al._Universal definition and classification of heart failure: a report of the Heart Failure Society of America, Heart Failure Association of the European Society of Cardiology, Japanese Heart Failure Society and Writing Committee of the Universal Definition of Heart Failure European Journal of Heart Failure (2021)

\section{O papel dos peptídeos natriuréticos no diagnóstico de insuficiência cardíaca}

Os peptídeos natriuréticos (NPs) são substâncias produzidas em contexto de sobrecarga de pressão e/ou volume. Em pacientes com IC aguda são utilizados para inferir elevadas pressões de enchimento das câmaras cardíacas, sendo utilizados para diagnóstico e prognóstico [11]. São produtos exclusivamente produzidos por cardiomiócitos por distensão celular (e também sinalização por citocina IL-1 $\beta$ e TNFa por exemplo - no caso do BNP) promovendo natriurese, vasodilatação, relaxamento e redução da fibrose miocárdica. Para a adequada compreensão é importante enfatizar que os NPs não são biomarcadores exclusivos da função sistólica do VE, mas de inúmeras anormalidades cardíacas funcionais e/ou estruturais, como, por exemplo, disfunção VD - em suma - qualquer processo de tensão que leve a estiramento e distensão de cardiomiócitos levará a secreção de NPs. Estudos com objetivo de avaliar acurácia diagnóstica dos NPs comparando o seu desempenho com a referência padrão para diagnóstico de IC tem encontrado de maneira consistente que os NPs possuem alta acurácia diagnóstica para IC. Adicionalmente, melhoram a acurácia do julgamento clínico no departamento de emergência. Os peptídeos natriuréticos apresentam alta capacidade de afastar o diagnóstico (alto valor preditivo negativo), na presença de níveis séricos de BNP $<100 \mathrm{pg} / \mathrm{mL}$ e NT-proBNP $<300 \mathrm{pg} / \mathrm{mL}$. Em decorrência dos vários fatores clínicos que podem estar associados a elevação dos peptídeos natriuréticos, reduzindo seu valor preditito positivo, níveis séricos intermediários (zona cinzenta) de BNP ou NT-proBNP necessitam da correlação clínica para confirmação do diagnóstico de IC aguda. Aproximadamente $20 \%$ dos pacientes com dispneia aguda apresentarão NPs na zona cinzenta, e $50 \%$ desses pacientes terão IC aguda como diagnóstico. O médico deverá considerar outros diagnósticos na ausência de características clínicas típicas e/ou específicas de IC. Em situações de rápida instalação, como edema agudo pulmonar flash, na insuficiência mitral aguda, ou no cor pulmonale agudo, podemos observar níveis não elevados dos NPs. A princípio não há diferença entre o uso do BNP ou do NT-proBNP exceto em pacientes em uso do medicamento sacubitril-valsartana (nome comercial Entresto®). A inibição da neprilisina pelo sacubritil promove elevação nos níveis séricos do BNP, tornando o NT-proBNP o exame de escolha. Abaixo encontra-se resumida as principais orientações para o uso:

- Sempre devem ser utilizados em conjunto com os sinais e/ou sintomas documentados na avaliação clínica.

- A sua medida deve ser realizada em todos os pacientes no departamento de emergência com sintomas sugestivos de IC, possuindo alta acurácia em diferenciar IC de outras causas de dispneia. Quanto maior o valor dos peptídeos natriuréticos, maior será a probabilidade que IC seja a causa dos sinais/sintomas do paciente.

- Os valores a partir dos quais os NPs indicam diagnóstico de IC são claramente maiores para IC aguda em relação a IC crônica.

- Existem diversos tipos de NPs, sendo o BNP e NT-proBNP os mais utilizados na prática médica, ambos possuindo acurácia diagnóstica e prognóstica comparável.

- Os resultados obtidos devem ser sempre interpretados considerando a função renal e índice de massa corporal (IMC), os dois principais interferentes em seus valores. Em pacientes obesos, recomenda-se utilizar valor de referência $50 \%$ menor.

- Em pacientes com marcadores de hipoperfusão tecidual, ou seja, choque, os NPs não podem ser utilizados para identificar 
Recomendação dos valores para diagnóstico de IC aguda

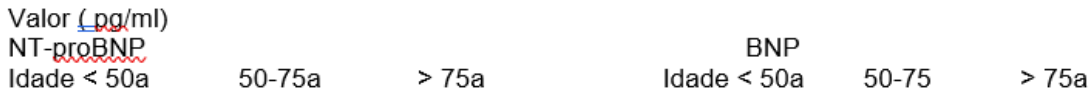

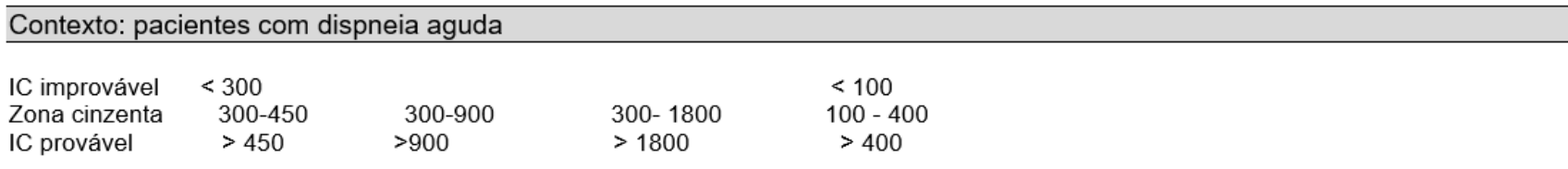

\section{Causas de NPs elevados que não IC}

Causas cardiovasculares: síndrome coronariana aguda, infarto agudo do miocárdio, embolia pulmonar, miocardite, cardiomiopatia hipertrófica, doença cardíaca valvar, doença cardíaca congênita, arritmias atriais ou ventriculares, contusão cardíaca, infiltração ou malignidade, cardioversão, choque, procedimentos cirúrgicos ou invasivos envolvendo o coração, hipertensão pulmonar, insuficiência de ventrículo direito, cardiomiopatias infiltrativas

Causas não-cardiovasculares: idade avançada, doença renal, doenças críticas (ex., sepse), acidente vascular cerebral isquêmico ou hemorrágico, doenças pulmonares ( ex., pneumonia, DPOC), doença hepática, anemia grave, anormalidades hormonais e metabólicas graves (ex., tireotoxicose, cetoacidose diabética, grandes queimados )

Tabela 2. Adaptado de Mueller et al Heart Failure Association of the European Society of Cardiology practical guidance on the use of natriuretic peptide concentrations 2019

a causa, como, por exemplo, choque cardiogênico/misto versus choque séptico.

Para consultar os valores de acordo com a idade e outras condições que interferem na medida dos peptídeos natriuréticos, vide Tabela 2.

\section{Avaliando a congestão na insuficiência cardíaca aguda}

Congestão é a principal razão que leva ao atendimento no departamento de emergência de pacientes com IC aguda, independente do fator precipitante. A congestão na insuficiência cardíaca é definida como o acúmulo de fluido no compartimento intravascular e no espaço intersticial, resultante do aumento das pressões de enchimento das câmaras cardíacas causadas por retenção inadequada de sódio e água pelos rins [13]. A cascata clássica da congestão (Figura 2) [4] se inicia por estágios subclínicos caracterizados por aumento das pressões de enchimento das câmaras cardíacas e pressões venosas (congestão hemodinâmica), seguido pela redistribuição para os pulmões e outros órgãos (congestão orgânica) e, finalmente, chegando ao surgimento de sinais e sintomas (congestão clínica). A congestão hemodinâmica pode preceder a congestão clínica em até semanas com uma relativa ausência de sintomas. Embora a congestão orgânica e clínica geralmente siga a congestão hemodinâmica, a correlação entre a pressão hidrostática e a formação de edema é fraca. Consequentemente, a formação de edema intersticial pode ocorrer mesmo na presença de pressões hidrostáticas discretamente elevadas. A congestão pode estar presente predominantemente no sistema vascular (congestão intravascular) ou no interstício (congestão tecidual), embora a maioria dos pacientes tenha uma combinação de congestão intravascular e tecidual (figura 3) [13]. Embora a expansão absoluta da volemia seja a base da congestão em muitos, senão na maioria dos pacientes com IC aguda, a redistribuição da volemia (expansão relativa) presente em grandes leitos venosos, como, por exemplo, a circulação venosa do compartimento abdominal, pode desempenhar um papel importante, sobretudo nos pacientes nos quais a descompensação ocorra rapidamente [13]. A congestão intravascular e a congestão tecidual podem ser identificadas $e$ diferenciadas com o uso de avaliações diagnósticas específicas, como exame físico, biomarcadores e técnicas de imagem [13].

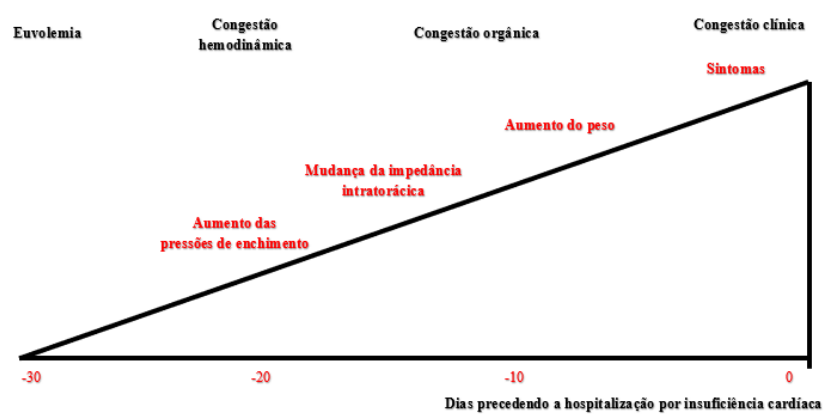

Figura 2. Adaptado de Arrigo et al . Optimising Heart Failure Therapies in the Acute Setting 2018. A figura demonstra a cascata da congestão tecidual. Observase que a congestão hemodinâmica causada pelo 
aumento das pressões de enchimento antecede em semanas os sintomas.

\section{Congestão tecidual}

Os pacientes com predomínio de congestão tecidual apresentam aumento gradual nas pressões de enchimento das câmaras cardíacas e da pressão venosa [13]. Entretanto, outros fatores contribuem para o fenótipo edemigênico presente neste tipo de congestão,como, por exemplo, a reabsorção crônica de sódio através dos túbulos renais na IC crônica e sua relação com os glicosaminoglicanos (GAG's) do interstício[13], possivelmente explicando a relação frágil entre pressão hidrostática e formação de edema. Devido às características dos fatores relacionados a este padrão de congestão, observa-se um curso clínico marcado por formação lenta e gradual de edema periférico, edema pulmonar e ascite. Esses pacientes geralmente são tratados com início ou intensificação da terapia com diuréticos. Entretanto, existem evidências questionando o papel de outras possíveis terapias.

\section{Congestão intravascular}

Os sintomas e sinais mais comuns de IC aguda crônica descompensada estão diretamente relacionados à congestão intravascular que pode resultar da expansão absoluta da volemia por meio de mecanismos interdependentes ou por rápida redistribuição central do volume intravascular da circulação venosa periférica ou esplâncnica [13]. Pacientes com predomínio de congestão intravascular se apresentam com aumento agudo da pressão arterial sistêmica, ocasionando o aumento repentino das pressões de enchimento das câmaras cardíacas e da pressão venosa. Esses pacientes geralmente respondem bem ao tratamento com vasodilatadores [13].

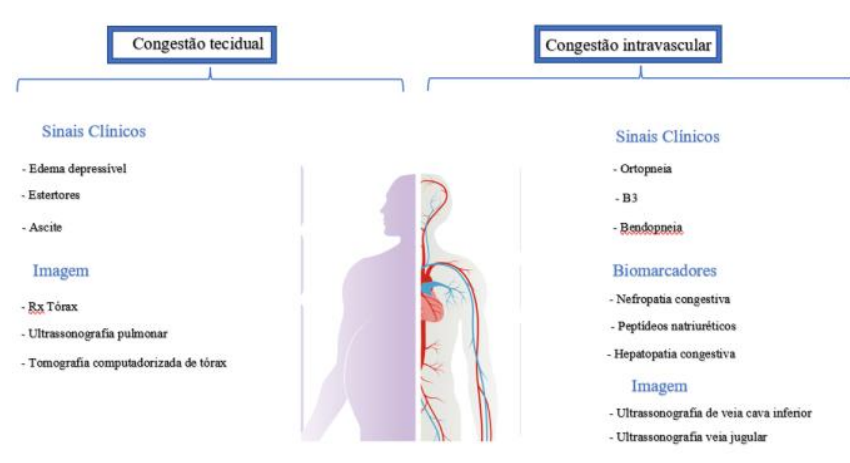

Figura 3. Adaptado de Boorsma et al Congestion in heart failure: a contemporary look at physiology, diagnosis and treatment 2020. Diferenças hipotetizadas entre os padrões de congestão tecidual e intravascular. A figura mostra os sinais clínicos, biomarcadores e métodos de imagem para caracterização do padrão de congestão. A nefropatia congestiva e hepatopatia congestiva são consequências diretas da elevada pressão venosa central. Alguns estudos têm demonstrado que a piora de função renal só é preditiva de desfecho cardiovascular ruim quando associada a congestão persistente. A elevação de enzimas hepáticas são marcadores independentes de prognóstico adverso. Bendopneia é a ocorrência de aumento da dispneia ao inclinar-se para a frente e está associada ao aumento das pressões de enchimento das câmaras cardíacas. A presença de bendopneia se correlaciona com a presença de ortopneia e intolerância ao exercício, mas não com estertores ou edema periférico, sendo um forte indicativo de congestão intravascular, em vez de edema tecidual. Esta noção é apoiada pela observação de que a presença de bendopneia se correlaciona com doença mais avançada em pacientes com hipertensão arterial pulmonar. A ortopneia é o resultado do aumento do fluxo sanguíneo venoso dos membros inferiores quando o paciente está em uma posição supina ou semi- supina, o que aumenta a pré-carga cardíaca. A liberação de peptídeos natriuréticos como marcador de sobrecarga de pressão/ volume é correlacionada a pressão de congestão intravascular ao invés de tecidual. A ultrassonografia da veia jugular pode ser uma medida mais precisa e reprodutível do que a inspeção visual de sua distensão. Esta abordagem mostrou se correlacionar bem com o estado de congestão e ser um preditor de reinternação por insuficiência cardíaca.

\section{Ultrassonografia point of care para avaliação objetiva de congestão pulmonar e sistêmica}

A definição universal de IC publicada pela ESC em 2021 exige que para o diagnóstico os sinais e/ou sintomas apresentados pelo paciente sejam corroborados por níveis elevados de peptídeos natriuréticos ou evidência objetiva de congestão pulmonar e/ou sistêmica. A detecção de congestão através do exame físico e/ou radiografia de tórax carece de sensibilidade suficiente para serem utilizados como únicas ferramentas $[8,9,10,11,13]$. A 
razão pela qual a ultrassonografia tem ganhado destaque na avaliação de pacientes com IC baseia-se em suas características:

- Exame não invasivo, seguro e rápido.

- Não há necessidade de mobilização do paciente.

- Desprovido de radiação.

- Necessidade de treinamento mínimo para reproduzir resultados homogêneos.

- Possibilidade de ser utilizado inúmeras vezes conforme necessidade da equipe assistencial, permitindo acompanhar a evolução da doença e identificar diagnósticos diferenciais ameaçadores à vida e que exigem tratamento rápido e acurado, como, por exemplo, tamponamento cardíaco.

- Os seus achados estão associados ao risco de hospitalização e morte no momento da alta do departamento de emergência.

O pulmão pode ser avaliado através da ultrassonografia para detecção de diversões padrões de acometimento com sensibilidade, especificidade e acurácia adequada. A detecção de múltiplos e difusos artefatos de reverberação (linhas - B; figura 4 e 5) são correlacionados com o conteúdo de água no interstício pulmonar e definem congestão em contexto apropriado. Estudos já demonstraram que a ultrassonografia pulmonar apresenta maior acurácia diagnóstica de IC aguda em pacientes que se apresentam com dispneia indiferenciada no departamento de emergência quando comparada a avaliação clínica tradicional e radiografia de tórax [9]. A ultrassonografia também pode auxiliar o médico em identificar os pacientes de maior risco de evolução desfavorável [10]. A quantificação de linhas -B no momento da alta em comparação à admissão está associada a maior risco de hospitalização e morte durante o período da fase vulnerável e independente de outras variáveis clínicas importantes [10].

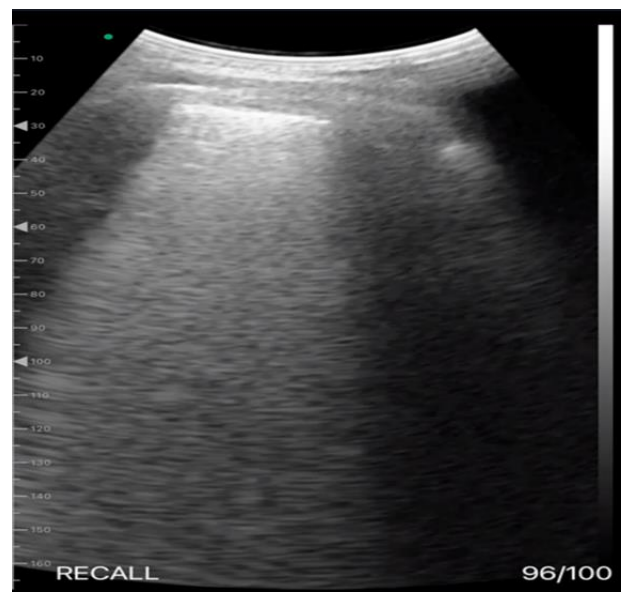

Figura 4. Ultrassonografia pulmonar demonstrando linhas hiperecogênicas oriundas da linha pleural, confluentes, que se estendem até a periferia da imagem e são dinâmicas com a respiração. A confluência de linhas $B$ indica $>5$ linhas por espaço examinado. Fonte: Cortesia de Dr. Geovane Wiebelling da Silva - médico assistente do prontosocorro do Hospital das Clínicas da FMUSP

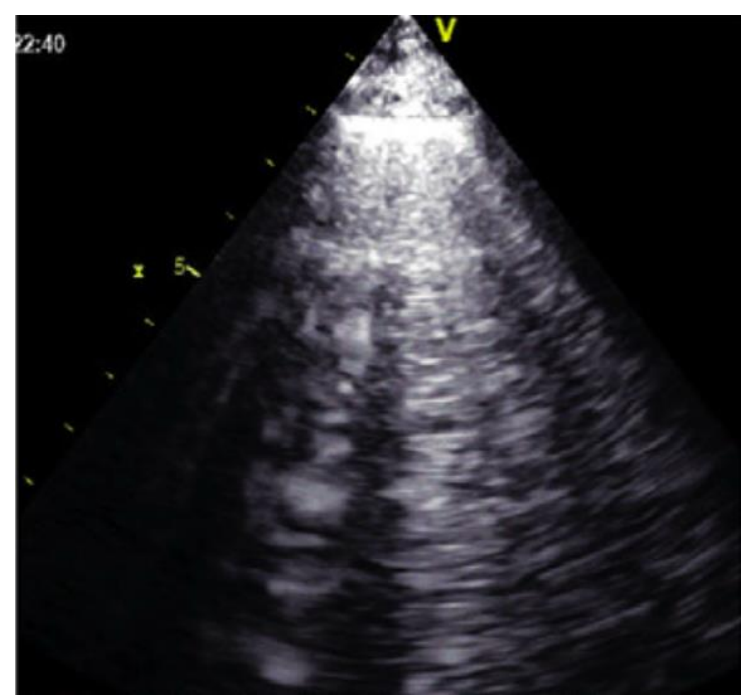

Figura 5. Ultrassonografia pulmonar demonstrando $>3$ linhas B não confluentes. Fonte: Cortesia de Dr.Geovane Wiebelling da Silva - médico assistente do pronto-socorro do Hospital das Clínicas da FMUSP
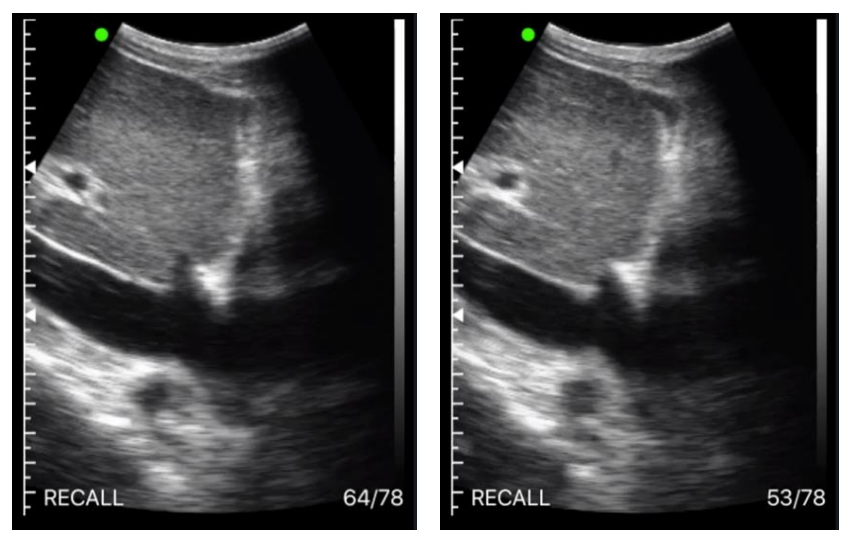

Figura 6 e 7. Ultrassonografia point of care de veia cava inferior em paciente respirando espontaneamente. Observa-se na imagem à esquerda uma veia cava pletórica na expiração com diâmetro $>2.1 \mathrm{~cm}$, enquanto à direita observa-se colapsibilidade $<=50 \%$ na inspiração. Fonte: Cortesia de Dr. Geovane Wiebelling da Silva - médico assistente do pronto-socorro do Hospital das Clínicas da FMUSP.

Como ferramenta caracteristicamente sensível, mas de baixa especificidade, a avaliação do diâmetro da veia cava inferior ( $\mathrm{VCl}$; figura 6$)$ e a 
magnitude de sua colapsibilidade respiratória (figura 7) através da ultrassonografia permite inferir a pressão venosa central e, consequentemente, as pressões de enchimento do ventrículo direito [9]. É importante ressaltar que esta informação diz respeito a pacientes respirando espontaneamente. A presença de uma veia cava inferior com diâmetro $>2.1 \mathrm{~cm}$ e colapsibilidade $<=50 \%$ define congestão sistêmica em contexto apropriado [9].

\section{A fase vulnerável após hospitalização por IC aguda}

O período pós-alta que está imediatamente após a hospitalização é o de maior risco para eventos adversos [1,2,4,7,8,13,14], sendo denominado fase vulnerável. A duração exata durante a qual a fase vulnerável se estende não é clara, mas dados sugerem duração de 2 a 3 meses. Importante ressaltar que reinternação após a fase vulnerável está associada a evolução natural da doença ocasionada pelo remodelamento cardíaco progressivo.

No geral, os pacientes hospitalizados por IC aguda morrem e são reinternados por diversos motivos, sejam cardíacos ou não. No entanto, para a grande maioria de pacientes com desfechos cardiovasculares ominosos pós-alta, particularmente reinternação, a congestão residual persistente no momento da alta é um fator crítico na fisiopatologia subjacente. A congestão clínica presente no atendimento geralmente melhora rápida $e$ dramaticamente com a terapia instituída, e a maioria dos pacientes apresentam sinais e sintomas mínimos ou ausentes na alta, indicando que a avaliação tradicional guiada por sinais e/ou sintomas não é capaz de identificar os pacientes elegíveis para prosseguir os cuidados fora do hospital. A alta do paciente na presença de congestão residual está associada a maior mortalidade e reinternação hospitalar por insuficiência cardíaca. Pacientes com congestão residual no dia $7^{0}$ de hospitalização têm um aumento de mais de duas vezes na mortalidade em 180 dias e um aumento de quase duas vezes no risco de reinternação por insuficiência cardíaca em comparação com pacientes sem congestão $[7,10,13]$.No estudo EVEREST os pacientes que apresentavam baixos scores de congestão clínica (aproximadamente $80 \%$ dos pacientes) apresentavam valores elevados de peptídeos natriuréticos, que como já explicado, estão correlacionados a congestão hemodinâmica. A mortalidade e 0 risco de reinternação para esses pacientes alcançou aproximadamente $46.9 \%$ em um seguimento médio de até 9.9 meses. A discordância frequente entre congestão clínica e valores de peptídeo natriurético é consistente com congestão hemodinâmica persistente. Apesar da associação da redução do valor dos peptídeos natriuréticos entre o momento do início da terapia de descongestão e o momento da alta com redução do risco de reinternação e mortalidade, os resultados são conflitantes para realizar uma recomendação baseada em valores.

A terapia de descongestão deve ter como objetivo alcançar euvolemia. Entretanto, é notório os efeitos em diversos sistemas orgânicos quando iniciada a estratégia de descongestão. Os efeitos potenciais da terapia de descongestão agressiva podem ser visualizadas através de exames laboratoriais comumente realizados na rotina diária, como, por exemplo, hemoconcetração levando inadvertidamente a modificações do planejamento. Não é incomum ocorrer hemoconcentração e aumento transitório da creatinina, indicando redução da volemia plasmática circulante e , por conseguinte, piora da função renal [19]. Entretanto, é importante lembrar que a congestão venosa parece ser o fator hemodinâmico mais importante como causa da piora da função renal em pacientes com IC aguda [20]. Em conformidade com melhora clínica, a estratégia de descongestão agressiva não deveria ser interrompida a despeito de piora da função renal.

É importante reconhecer os fatores clínicos adicionais que tendem a estar associados a piores desfechos: hiponatremia, piora da função renal associada a congestão, hipotensão (particularmente intolerante a terapia médica relacionada a mudança de prognóstico), anemia, peptídeo natriurético persistentemente elevado e dissincronia ventricular [14]. Embora estes possam não ser alvos terapêuticos, presença de qualquer um desses fatores no momento de alta deve levar ao planejamento de acompanhamento rigoroso para evitar o risco de reinternação durante a fase vulnerável. Embora esta revisão não tenha como foco as opções terapêuticas agudas ou crônicas, é essencial destacar a importância de otimizar a terapia médica relacionada a mudança de prognóstico.

Abaixo, resumimos as principais recomendações que interferem positivamente na fase vulnerável:

1. Todos os pacientes devem ser avaliados em busca de congestão residual. $O$ uso do POCUS e peptídeos natriuréticos podem indicar descongestão ineficaz. A melhora dos 
sintomas de congestão não são confiáveis em identificar descongestão eficaz.

2. Todos os pacientes com ICFER devem ter terapia relacionada a mudança de prognóstico iniciadas e/ou otimizadas antes da alta. A alta sem prescrição de inibidores do sistema renina-angiotensina e/ou betabloqueadores está associado com alto risco de readmissão $[15,16]$.

3. A digoxina em pacientes com ICFER apresenta capacidade de reduzir readmissão a despeito do efeito neutro em reduzir mortalidade. O seu uso não é indicado em ICFEP [16].

4. O paciente deve ter atendimento agendado em até 1-2 semanas da alta para avaliar congestão residual, avaliar tolerância a medicações orais e otimizar terapia relacionada a mudança de prognóstico $[15,16]$.

5. Carboximaltose férrica deve ser considerada para deficiência de ferro, definida por ferritica sérica $<100 \mathrm{ng} / \mathrm{ml}$ ou ferritina sérica $100-$ $299 \mathrm{ng} / \mathrm{ml}$ com saturação de transferrina menor que $20 \%$ para reduzir sintomas e reduzir readmissão [17].

6. O uso de inibidor do co-transportador de sódio-glicose 2 ( SGLT2) deve ser considerado em pacientes com ICFER para reduzir mortalidade e hospitalização [18].

\section{Conclusão}

A IC aguda é um problema de saúde de magnitude epidêmica e crescente. No Brasil e em regiões de média a baixa renda, a calamidade social soma-se às práticas médicas desprovidas de evidência. O departamento de emergência como porta de entrada deve estar alinhado com as medidas que comprovadamente possam ser iniciadas e/ou intensificadas para reduzir a mortalidade e o risco de reinternações por IC aguda. A melhor compreensão sobre a fisiopatologia da congestão e sua avaliação integrada desponta como alicerce para melhorar as intervenções realizadas. Os dados dos estudos realizados até 0 momento permitem que 0 médico emergencista possa atuar para reduzir a mortalidade e risco de reinternação durante a fase vulnerável. A compreensão dos problemas do sistema de saúde que se manifestam como resultados ruins para pacientes hospitalizados ou simplesmente avaliados e tratados no departamento de emergência por IC é vital para o desenvolvimento de intervenções bemsucedidas

\section{Referências}

1. Diretriz Brasileira de Insuficiência Cardíaca Crônica e Aguda. Arq Bras Cardiol. 2018; 111(3):436-539.

2. Arrigo M, Jessup M, Mullens W, Reza N, Shah AM, Sliwa Ket al . Acute heart failure Nature Reviews Disease Primers (2020)

3. Universal definition and classification of heart failure: a report of the Heart Failure Society of America, Heart Failure Association of the European Society of Cardiology, Japanese Heart Failure Society and Writing Committee of the Universal Definition of Heart Failure . European Journal of Heart Failure (2021) 23, 352-380

4. Arrigo $M$, Nijst $P$ and Rudiger $A$. Optimising Heart Failure Therapies in the Acute Setting . Cardiac Failure Review 2018;4(1):38-42

5. Albuquerque DC, Neto JD, Bacal F, Rohde LE, Bernardez-Pereira $S$, Berwanger $O$, et al; Investigadores Estudo BREATHE. I Brazilian Registry of Heart Failure - Clinical aspects, care quality and hospitalization outcomes. Arq Bras Cardiol. 2015;104(6):433-42.

6. Dokainish, $\mathrm{H}$. et al. Global mortality variations in patients with heart failure: results from the International Congestive Heart Failure (INTERCHF) prospective cohort study. Lancet Glob. Health 5, e665-e672 (2017).

7. Greene SJ, Fonarow GC, Vaduganathan M, Khan SS, Butler $J$ and Gheorghiade. The vulnerable phase after hospitalization for heart failure Nat. Rev. Cardiol2015

8. Girerd N, Seronde MF, Coiro S, Chouihed T, Bilbault $P$, Braun $F$ et al. Integrative Assessment of Congestion in Heart Failure Throughout the Patient Journey J Am Coll Cardiol HF 2018;6:273-85

9. Pivetta E, Goffi A, Lupia E, Tizzani M, Porrino $G$, Ferreri $E$ et al. Lung UltrasoundImplemented Diagnosis of Acute Decompensated Heart Failure in the ED CHEST 2015; 148(1):202-210

10. Platz E, Campbell RT, Clagget B, Lewis EF, Groarke JD, Docherty KF et al . Prevalence of Pulmonary Congestion and Short- and LongTerm Outcomes J Am Coll Cardiol HF 2019;7:849-58 
11. Heart Failure Association of the European Society of Cardiology practical guidance on the use of natriuretic peptide concentrations. European Journal of Heart Failure (2019) 21, 715-731

12. Matsue $\mathrm{Y}$, Damman K, Voors AA, et al. Timeto-furosemide treat- ment and mortality in patients hospitalized with acute heart failure. J Am Coll Cardiol 2017;69:3042-51.

13. Boorsma EM, Maaten JMT, Damman K, Dinh Wilfried, Gustafsson Finn, Goldsmith $S$ et al . Congestion in heart failure: a contemporary look at physiology, diagnosis and treatment Nature Reviews Cardiology volume 17, pages 641-655 (2020)

14. Njoroge JN , Teerlink JR . Pathophysiology and Therapeutic Approaches to Acute Decompensated Heart Failure Circulation Research. 2021;128:1468-1486

15. McDonagh TA, et al. 2021 ESC Guidelines for the diagnosis and treatment of acute and chronic heart failure: Developed by the Task Force for the diagnosis and treatment of acute and chronic heart failure of the European Society of Cardiology (ESC) With the special contribution of the Heart Failure Association (HFA) of the ESC. European Heart Journal

16. R.C. Bourge, J.L. Fleg, G.C. Fonarow, et al. Digoxin reduces 30-day all-cause hospital admission in older patients with chronic systolic heart failure, Am. J. Med. 126 (8) (2013) 701-708.

17. Ponikowski P, Kirwan BA, Anker SD, McDonagh $\mathrm{T}$, Dorobantu M, Drozdz J et al . AFFIRM- AHF Investigators. Ferric carboxymaltose for iron deficiency at discharge after acute heart failure: a multicentre, doubleblind, randomised, controlled trial. Lancet 2020; 396:1895 - 1904.

18. JJV McMurray et al. Dapagliflozin in Patients with Heart Failure and Reduced Ejection Fraction. N Engl J Med 381 (21), 1995-2008. 2019.

19. Testani J, Chen J, McCauley B, Kimmel S, Shannon R. Potential Effects of Aggressive Decongestion During the Treatment of Decompensated Heart Failure on Renal Function and Survival Circulation. 2010;122:265-272

20. Mullens W, Abrahams Z, Francis G, Sokos G, Taylor D, Starling $\mathrm{R}$ et al . Importance of Venous Congestion for Worsening of Renal
Function in Advanced Decompensated Heart Failure JACC Vol. 53, No. 7, 2009:589 -96

\section{Informações sobre o Artigo}

Recebido: 20/12/2021

Aceito: 30/12/2021

Conflitos de interesse: nenhum.

Agências financiadoras: nenhuma. 